Research Article

\title{
Application of Blockchain-Based Sports Health Data Collection System in the Development of Sports Industry
}

\author{
Song Yu \\ School of Economics and Management, Shanghai University of Sport, Shanghai 200438, China \\ Correspondence should be addressed to Song Yu; songyu@sus.edu.cn
}

Received 8 May 2021; Revised 24 May 2021; Accepted 31 May 2021; Published 10 June 2021

Academic Editor: Sang-Bing Tsai

Copyright (C) 2021 Song Yu. This is an open access article distributed under the Creative Commons Attribution License, which permits unrestricted use, distribution, and reproduction in any medium, provided the original work is properly cited.

In recent years, with the continuous development of blockchain technology and the prosperity of digital currency, various industries have carried out extensive practice around the application of blockchain technology, such as my country's sports industry. Through the measurement and collection of athletes' health and exercise information, the athlete's exercise volume and exercise level can be quantitatively evaluated. Blockchain technology provides a new opportunity for the development of the sports industry. The characteristics of the decentralization, security, credibility, and traceability of the data collection system are highly compatible with the development of the sports industry. In order to develop and expand the sports industry and scientifically evaluate the physical fitness of athletes, this research focuses on the blockchain-based sports health data collection system in the sports industry. This article adopts the accuracy index and the formula of data return and conducts demand analysis, accuracy, and recall analysis of the health data collection system. The research conclusion of this paper is that when using sensors to collect sports health data, the data collection of sensors located on the arm is more accurate, with an accuracy rate of over $90 \%$, and the collection effect of multinode sensors is higher than that of single-node sensors. The collection of sports health data is beneficial to the training of athletes and the development of the sports industry to meet the growing demand for sports health. Our country's sports industry will also develop with the application of blockchain.

\section{Introduction}

In order to effectively formulate training plans and avoid injuries and illnesses, it is essential to monitor athletes' health data during training and competitions. One of the key issues is how to reliably collect important data from them in real time. Blockchain is a segmented database system with multiple independent nodes participating together and is jointly maintained by these nodes. The information processed by it is highly secure and the process is efficient and transparent. Based on the characteristics of blockchain distributed storage and decentralization, data systems based on this technology can efficiently collect information and apply it to the development of other industries. At present, the sports industry urgently needs to change the development situation, emphasizing the construction of a platform for information exchange and the application of information technology, and blockchain technology can help the sports industry expand its development space and revitalize the sports market. The sports health data collection system based on the blockchain in the sports industry is a development opportunity for the sports industry.

In view of the above background, many scholars at home and abroad have conducted relevant research. From a security perspective, Kshetri highlighted that blockchainbased solutions can outperform the current IoT ecosystem in many aspects. Through practical applications and practical examples, the author believes that the decentralized nature of the blockchain may cause malicious participants to be less sensitive to manipulation and forgery and considers how the blockchain-based identity and access management system responds to the security of the Internet of things. Some of the key challenges related in the study are that the author analyzed and described the role of blockchain in tracking 
insecure sources in the supply chain related to IoT devices in detail. He believes that the use of blockchain can be used to target security vulnerabilities in the Internet of Things. It is contained. This article makes a reasonable analysis of the role of blockchain but fails to describe its development prospects in some industries $[1,2]$. In his research, Bazi introduced a method of using location data to optimize road data collection vehicle routes. Many developed countries will conduct regular road testing, and the data obtained will be used as input to the road management system. At present, the transportation departments in many cities plan this data collection by providing a list of roads that must be tested and then leaving the routing of the vehicles to the equipment operators who usually perform the work in a temporary manner. This study proposes the process of coding a list of test roads, selecting the appropriate hotel in the test area, and applying the traveling salesman problem with hotel stops to derive the route. Applying these procedures to case studies shows that this road test method can save a lot of cost compared to current ad hoc methods. However, this study only describes the road test in detail, and its application is relatively limited [3]. Yang believes that the sports industry has shown great potential in improving the national economy, guiding social consumption, and adjusting the industrial structure. However, compared with the rapid development of the sports industry, the research on the growth law of the sports culture industry is relatively backward, which greatly restricts the further development of the sports industry. In response to this problem, the author analyzed the feasibility of forecasting the development of sports culture industry based on big data theory from the perspective of data mining and proposed a prediction model for the development of sports culture industry based on genetic neural network, which realized the development of sports culture industry. It is concluded that the optimized neural network can effectively improve the efficiency and accuracy of the prediction and has strong stability to the development law of the sports culture industry. The research predicts the sports culture industry but does not analyze the various quality indicators of athletes, which is not comprehensive enough [4].

This article studies the application of the blockchainbased sports and health data collection system in the development of the sports industry. First, it briefly introduces the advantages of the blockchain and the content of the data collection system, using the accuracy index and the formula of data backhaul. For the health data collection system, we conducted demand analysis, accuracy rate and recall rate analysis, and data packet success rate analysis and concluded that the sports health data collection is beneficial to the training of athletes and the development of the sports industry to meet the growing sports health. The needs of my country's sports industry will also develop with the application of blockchain. The innovation of this article is to apply the popular blockchain technology to the traditional data collection system and develop this technology to broaden the development market of the sports industry.

\section{Application Research Method of Sports Health Data Collection System Based on Blockchain in the Development of Sports Industry}

2.1. Blockchain. There are many types of sports health data collection systems, some are based on the Internet of Things, some are based on artificial intelligence, and some are based on databases. Blockchain is mainly composed of user layer, extension layer, and protocol layer. It has the characteristics of decentralization, openness, autonomy, nontamperable information, and anonymity $[5,6]$. These features enable each module in the blockchain to safely update or transmit its own data. This technology can save data to ensure reliability. In the case of information asymmetry, trust can be established without the supervision and approval of the central management agency, which greatly improves transaction efficiency and reduces transaction costs. At the same time, when the development of the sports industry uses confidential or private sports health data, blockchain can realize data sharing through remote technology [7]. The blockchain health data collection system does not require multiple levels of identity verification and provides access to data for everyone in the blockchain architecture. These functions can help solve the various challenges facing the development of the sports industry today.

2.2. Data Collection System. Generally speaking, a data acquisition system consists of three parts: sensors, data acquisition instruments, and computers. The data collector is mainly to obtain the data in the information equipment, supplemented by various other functions such as communication and diagnosis. The maximum configurable program combination module is convenient for data collection and human-computer interaction.

At present, the collection of sports health information has gone through several stages of development. In the network collection stage, electronic devices such as sensors are used to detect human-related information, and then the detected data is used to realize automatic collection and upload. The methods used to transmit data in portable devices include traditional wired transmission methods and current wireless transmission methods, such as $\mathrm{Wi}-\mathrm{Fi}$, Bluetooth, and ZigBee $[8,9]$. In recent years, researchers at home and abroad have conducted a lot of research and application in the field of motion detection using wireless technology and sensor technology, which has promoted the development of monitoring technology in the sports industry. The system collects user mobile and physical health information in real time, interacts with the cloud computing platform through blockchain encryption technology, performs data processing and professional diagnosis, and provides a scientific reference for using blockchain to establish remote real-time dynamic sports and fitness management technology model and basis [10, 11].

As shown in Figure 1, the health data collection system collects relevant information from athletes, such as various physical fitness indicators, and then converts such 


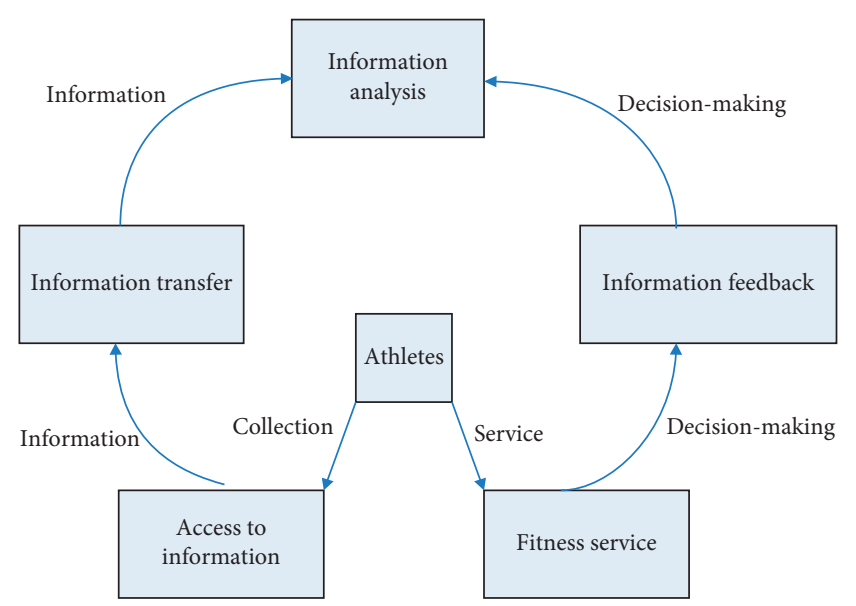

FIGURE 1: Schematic diagram of the information function.

information and finally conducts targeted analysis. Otherwise, they collect such information for customized health services and then get feedback from the athletes and finally perform the same analysis. People can monitor and evaluate their health progress in time based on these parameters and adjust the amount and intensity of exercise in time to make scientific exercise possible [12].

\section{Application Research Experiment of Sports Health Data Collection System Based on Blockchain in the Development of Sports Industry}

3.1. Accuracy Index. In order to reflect the accuracy of the sports health data collection system, precision and recall rate are used as evaluation indicators here. In this model, for the input test data, the model will get the data category. Accuracy refers to the proportion of samples that really belong to the category $\mathrm{Ci}$ among all the samples that the model judges to be $\mathrm{C} i$; the recall rate refers to the concentration in the data, the proportion of samples that are judged to be correct $[3,13]$. In the binary classification problem, assuming that the positive sample set output by the model is $A$, and the actual positive sample data set is $B$, then the formula for precision and recall is as follows:

$$
\begin{aligned}
& P=\frac{|A \cap B|}{|A|}, \\
& R=\frac{|A \cap B|}{|B|} .
\end{aligned}
$$

The accuracy rate represents the proportion of data samples that are correctly classified. Assuming that the total number of samples in the data domain is $n$, for sample $i$, the predicted category label is the true category label; then the accuracy rate can be defined as follows:

$$
A=\frac{\sum_{i=1}^{n} l\left(y_{i}=Y_{i}\right)}{n} .
$$

Among them, $l(x)$ is the indicator function. The larger the accuracy rate, the closer the indicated number, that is, the more accurate the overall sample prediction.

3.2. Data Backhaul. In the data processing and transmission model, the data backhaul is mainly analyzed. The link from the user side to the computing server side uses the millimeter wave frequency band $[14,15]$. Millimeter wave communication generally refers to a communication method that uses millimeter waves with a frequency band of 30 to 300 gigahertz and a wavelength of 1 to $10 \mathrm{~mm}$ as a carrier to transmit data: its beam forming method, high frequency band, less interference, stable and reliable transmission, and strong penetrating ability to sand, smoke, and dust $[16,17]$. Because millimeter wave communication is greatly affected by outdoor weather, this fading problem is not the focus of this research. In a communication system, the signal receiver has a line of sight only when there are no visible obstacles in the transmission link between the signal transmitter and the signal receiver. When passing an intermediate obstacle, there is no line of sight between the signal transmitter and the signal receiver $[18,19]$.

Generally, at a specified location, the path loss is expressed in $\mathrm{db}$ as follows:

$$
P L=P_{T X}-P_{R X}+G_{T X}+G_{R X} .
$$

Among them, $P_{T X}$ represents the total transmit power, $P_{R X}$ represents the total received power, $G_{T X}$ represents the transmit antenna, and $G_{R X}$ represents receive antenna gain. Path loss is also called propagation loss, that is, the loss caused by the propagation of radio waves in space, which is mainly determined by the radiation propagation of the transmit power. The transmission process has nothing to do with the characteristics of the transmitter and receiver, so the free space path loss described in $\mathrm{dB}$ is

$$
\bar{L}_{\text {Los }}(h)=32.5+20 \log _{10}(f)+10 \gamma \log _{10}(h)+A \times h .
$$

Among them, $f(\mathrm{MHz})$ represents the carrier frequency, and $\gamma$ represents the path loss index, and its value depends on the environment in the transmission path. $h$ represents the transmission distance between nodes, where the unit is $\mathrm{km}$; $A$ represents the attenuation coefficient of the atmosphere and rain. In the simulation, we make the transmission nodes on the backhaul link connect all viewpoints.

In the feature-to-result mapping, a layer of sigmoid function mapping is added to limit the predicted value to $[0$, $1]$, which can output the probabilities of different categories $[20,21]$. The probability $p(y=1 \mid x, \theta)$ indicates that the probability of $y$ is 1 when the characteristic variable $x$ is given and $(x)=p(y=1 \mid x, \theta)$, and then a logistic regression model is obtained:

$$
h_{\theta}(x)=\left[1+\exp \left(-\theta^{T} x\right)\right]^{-1} .
$$

Among them, $\theta=\left\{\theta_{1}, \theta_{2}, \ldots, \theta_{p}\right\}$ represents the coefficient value corresponding to each feature, the $\theta$ value. It can be obtained by solving the maximum likelihood estimation 
function [22]. Assuming that each sample in the data set is independent of each other, the likelihood function is

$$
I(\theta)=\prod_{i=1}^{n}\left[h_{\theta}(x)\right]^{y i} \cdot\left[1-h_{\theta}(x)\right]^{1-y_{i}}
$$

In practical applications, when classifying feature instances, we choose an instance with the largest probability value as the final category:

$$
y=f(x)=\arg \max \frac{\prod_{j=1}^{n} P\left(X^{(j)}=x^{(j)} / Y=c_{k}\right) P\left(Y=c_{k}\right)}{\sum_{l=1}^{K}\left(Y=c_{k}\right) \prod_{j=1}^{n} P\left(X^{(j)}=x^{(j)} / Y=c_{k}\right) .}
$$

A single data stream is mainly analyzed here. Assuming that the data collection end user's demand is $D$, it mainly depends on the user and the application used. In order to reflect the demand, it is assumed that the uncertainty and sensitivity of demand are inversely proportional $[23,24]$. We use the following formula to describe the relationship between them:

$$
D=(a-p) \cdot \frac{1}{b}
$$

\section{Application Research Analysis of Sports Health Data Collection System Based on Blockchain in the Development of Sports Industry}

4.1. Demand Analysis. From the perspective of demand, the sports health data collection system needs to regularly record various physical fitness indicators of athletes, including body mass index (BMI), heart rate, and basal metabolic rate (BMR). The specifics can be quantified to different Data obtained from distance running, long jump, squat, snatch and other items. Among them, BMI is an index used to judge whether the weight is normal. When the BMI is lower than 25, the risk of hypertension in Chinese people begins to increase. The basal metabolic rate refers to the minimum energy required to maintain various basic physiological activities in a state of awake, resting, fasting, and relaxed thoughts in a natural temperature environment. This is a system that is constantly updated over time. All registration information must ensure its authenticity and existence, and it can track the entire process of athlete growth. The purpose of designing this system is to establish a decentralized system, which can not only ensure the disclosure of athletes' own information but also prevent malicious tampering, which can improve the credibility of the sports industry and reduce the workload of related personnel, which will greatly contribute to the development of the sports industry.

4.2. Accuracy and Recall Analysis. Because current sensors have problems such as being greatly affected by the environment and inaccurate recognition actions, experiments are designed to place the sensors in different positions of the body to avoid randomness. In order to evaluate the accuracy of this type of data acquisition system, sensors were placed on the athlete's waist, left arm, right arm, left leg, and right leg, and the recall rate, precision, and accuracy formula mentioned in Section 3.1 were used to perform calculation. In addition, this part uses three different algorithms, among which KNN uses the method of measuring the distance between different feature values for classification, SVM is a supervised learning algorithm that can be used for classification and regression tasks, and the NCC normalized cross-correlation matching method is $A$ matching method based on image gray information.

It can be seen from Table 1 that the KNN algorithm can obtain a higher recognition accuracy rate as a whole, and the average accuracy rate of the arm part is higher than that of other parts. It can be seen that when a single sensor is used for human movement recognition, the hand plays the most important role.

It can be seen from Table 2 that both the KNN algorithm and the SVM algorithm have higher recognition performance, while the NCC algorithm has a certain gap with the other two. When calculating the accuracy, the accuracy of using SVM reached $96.9 \%$, the accuracy of using KNN algorithm was $98.2 \%$, and the accuracy of NCC was significantly lower than the other two, which was $94.6 \%$. Through the comparison of single-sensor node recognition and combined sensor node recognition, it can be seen that under the same algorithm, multinode combined recognition can achieve better results than single-node recognition.

4.3. Data Packet Success Rate Analysis. In order to evaluate the data packet success rate of the wireless important data collection system, we selected 30 members of a sports club to conduct experiments. It is assumed that the data packets successfully received on all the collection nodes will be forwarded to the personal computer of the coach through the collection node or a single hop. In this case, the data packet can be sent to the coach after successfully receiving at least one collection node.

As shown in Figure 2, for each given number of collection nodes, the collection node with the highest packet success rate is selected for combination, and the distance between collection nodes should be as equal as possible. It can be seen that in the $920 \mathrm{MHz}$ band, the use of more than three acquisition nodes does not improve the success rate of data packets. Whether using 2 nodes or more, the success rate is around 1.0; on the other hand, for the $2.4 \mathrm{GHz}$ band with the increase in the number of collection nodes, the grouping power will gradually increase. Using 6 collection nodes, a grouping power of about 0.8 can also be obtained.

Figure 3 shows the second half of the data. It can be clearly seen that in the $2.4 \mathrm{GHz}$ band, even if 6 acquisition nodes are used, the data packet success rate is only $80 \%$ and $20 \%$ is lost. It can be concluded that when the sensor band is 92.4 GHz, there is less data loss and fewer data nodes are used.

4.4. Application Analysis. The sports health data collection system based on blockchain technology will be widely used 
TABLE 1: Single-sensor node recognition.

\begin{tabular}{lcccc}
\hline Body parts & \multicolumn{3}{c}{ Accuracy } \\
& SVM $(\%)$ & KNN $(K=10)(\%)$ & NCC (\%) & 88.9 \\
\hline Waist & 89.5 & 89.9 & 92.9 & 93.4 \\
Left arm & 93.2 & 93.5 & 90.6 & 91.5 \\
Right arm & 91.4 & 92.5 & 88.8 & 89.5 \\
Left leg & 89.4 & 90.2 & 85.9 & 87.4 \\
Right leg & 87.7 & 88.5 & \\
\hline
\end{tabular}

TABle 2: Multisensor node recognition.

\begin{tabular}{lccc}
\hline Algorithm & Accuracy (\%) & Recall (\%) & Precision (\%) \\
\hline SVM & 97.6 & 96.1 & 96.9 \\
KNN $(K=10)$ & 97.4 & 95.9 & 98.2 \\
NCC & 92.9 & 92.7 & 94.6 \\
\hline
\end{tabular}

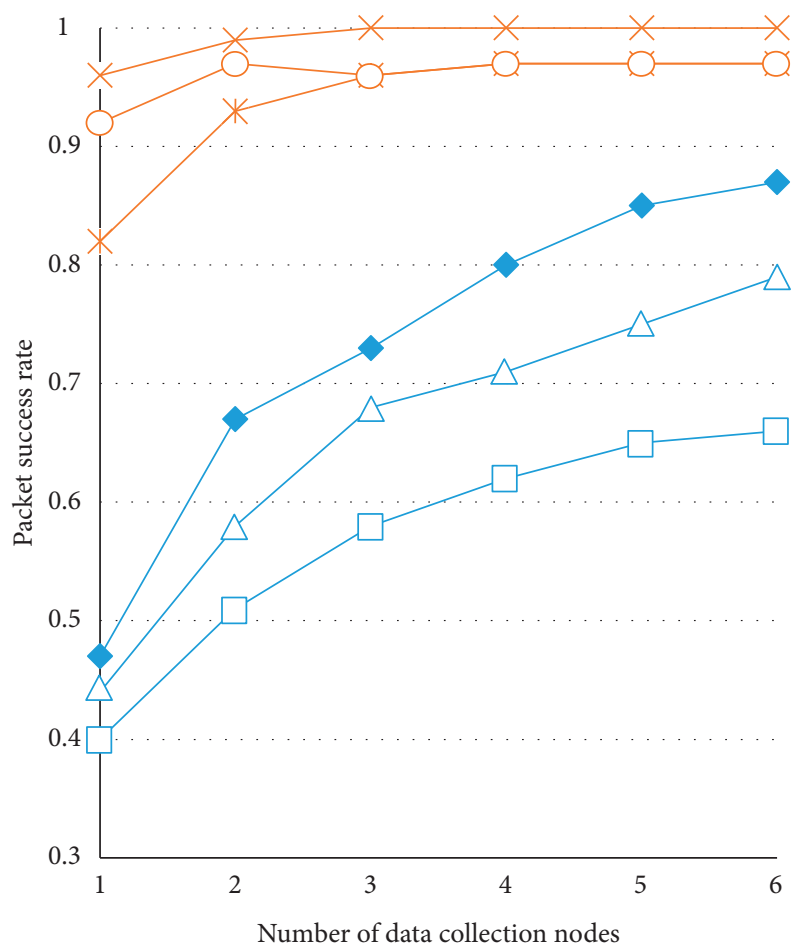

\begin{tabular}{|c|c|}
\hline $2.4 \mathrm{GHz}$ & $920 \mathrm{MHz}$ \\
\hline $\begin{array}{l}\text { Antenna height } \\
\text { diversity }\end{array}$ & $\begin{array}{l}-X-\begin{array}{l}\text { Antenna height } \\
\text { diversity }\end{array}\end{array}$ \\
\hline Antenna height $=1 \mathrm{~m}$ & $-X-$ Antenna height $=1 \mathrm{~m}$ \\
\hline Antenna height $=$ & - Antenna height $=2 \mathrm{~m}$ \\
\hline
\end{tabular}

FIgURE 2: First half of the packet success rate.

in the sports industry. First of all, the system can help athletes improve their existing training methods. Through the recording and collection of various indicators of their physical fitness, it can help coaches and sports departments understand the current status of athletes, reasonably arrange training intensity and frequency, and enable athletes to achieve scientific development efficiently. Similarly, this system can also help predict the future development trend of athletes, reduce the waste of human and material resources,

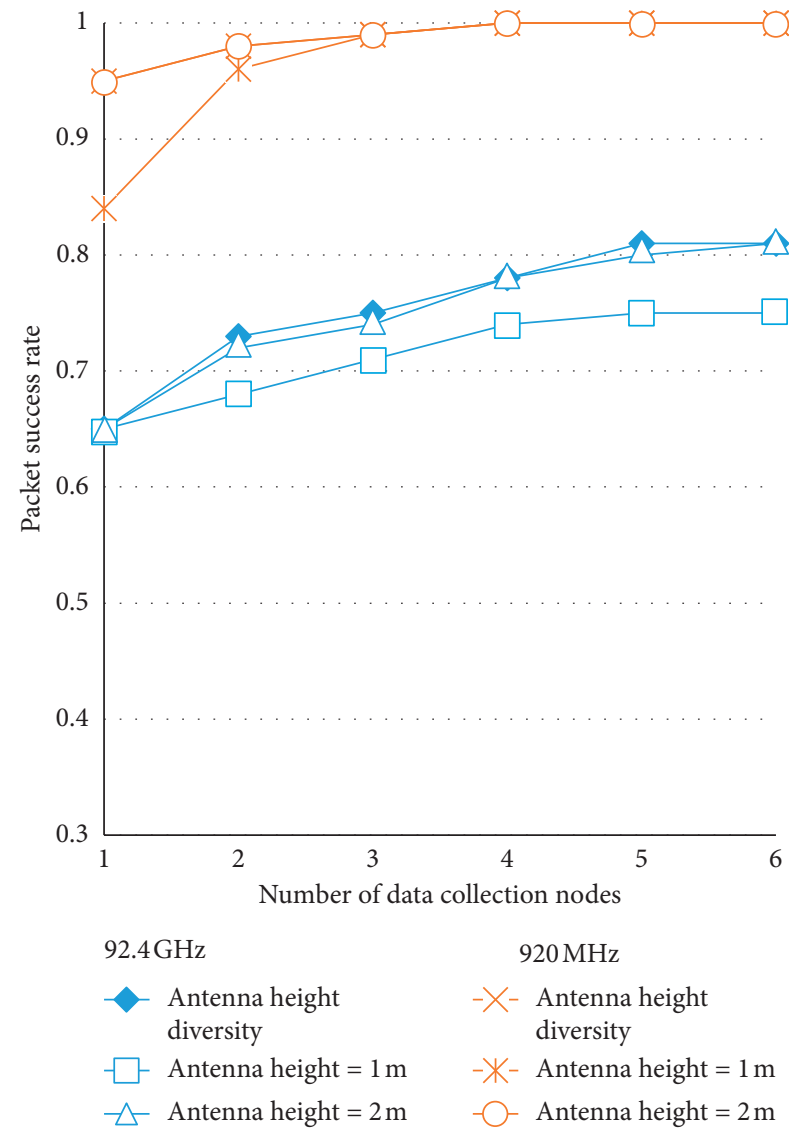

Figure 3: Second half of the packet success rate.

and play a key role in cultivating sports talents in the country.

Secondly, this data collection system can also help the sports industry develop business. For the control of sports health data, corresponding auxiliary products can be developed, such as the optimization design of sports shoes and clothing, the enhancement of the efficacy of health care drugs, and the actualization of the system for application in smart watches and other technological products, so that the public can also experience personalized customized services.

In addition, the centralization of my country's sports industry is relatively serious. Although sports lottery and other sports guessing industries and competitions occupy a large amount of bonuses, they lack control and are prone to illegal behavior. Such a data collection system can make transactions open and transparent and make the guessing game have a certain degree of predictability. 


\section{Conclusion}

The sports health data collection system can help understand the athlete's own condition, so as to better and target the training arrangements for the athletes. Due to the autonomy and decentralization of blockchain technology, various drawbacks in the sports industry can be that the use of blockchain technology is solved. The progress of sports health information collection has evolved from the traditional collection method to the ubiquitous collection method today. Various health and sports indicators of the human body are tested anytime and anywhere to provide a scientific basis for human health assessment. With the help of blockchain technology, the integrity of the sports industry system has been improved, and a new model for formulating sports health plans has been established, which is of great significance to the development and growth of the sports industry. At the same time, this article also has some shortcomings. The number of selected data sets is not large enough, and a specific sports health data collection system is not designed for simulation analysis. I hope that it can be continuously improved with in-depth learning in the future.

\section{Data Availability}

No data were used to support this study.

\section{Conflicts of Interest}

The author declares they no conflicts of interest.

\section{References}

[1] N. Kshetri, "Can blockchain strengthen the internet of things?" It Professional, vol. 19, no. 4, pp. 68-72, 2017.

[2] J. Chen, Z. Lv, and H. Song, "Design of personnel big data management system based on blockchain," Future Generation Computer Systems, vol. 101, pp. 1122-1129, 2019.

[3] G. Bazi, J. El Khoury, and F. J. Srour, "Integrating data collection optimization into pavement management systems," Business \& Information Systems Engineering, vol. 59, no. 3, pp. 135-146, 2017.

[4] K. Yang, "The construction of sports culture industry growth forecast model based on big data," Personal and Ubiquitous Computing, vol. 24, no. 1, pp. 5-17, 2020.

[5] I. M. Asif, "Celebrating the advancement of science in sports medicine: a 10-year collaboration between sports health and the amssm," Sports Health: A Multidisciplinary Approach, vol. 10, no. 2, pp. 110-112, 2018.

[6] J. Systems, "Map Gretel: social map service supporting a national mapping agency in data collection," Journal of Geographical Systems, vol. 21, no. 1, pp. 43-59, 2019.

[7] S. Underwood, "Blockchain beyond bitcoin," Communications of the ACM, vol. 59, no. 11, pp. 15-17, 2016.

[8] D. Kraft, "Difficulty control for blockchain-based consensus systems," Peer-to-Peer Networking and Applications, vol. 9, no. 2, pp. 397-413, 2016.

[9] D. Bradley and B. Hill, "Diversity and innovation in the FADN data collection systems in the EU-28," EuroChoices, vol. 15, no. 3, pp. 5-10, 2016.
[10] X. Xia, Y. Zhou, J. Li, and R. Yu, "Quality-aware sparse data collection in MEC-enhanced mobile crowdsensing systems," IEEE Transactions on Computational Social Systems, vol. 6, no. 5, pp. 1051-1062, 2019.

[11] J. Yang, J. Wen, B. Jiang, and H. Wang, "Blockchain-based sharing and tamper-proof framework of big data networking," IEEE Network, vol. 34, no. 4, pp. 62-67, 2020.

[12] R. Lou, W. Wang, X. Li, Y. Zheng, and Z. Lv, "Prediction of ocean wave height suitable for ship autopilot," IEEE Transactions on Intelligent Transportation Systems, vol. 2021, Article ID 3067040, 10 pages, 2021.

[13] H. Li, F. Guo, W. Zhang, J. Wang, and J. Xing, "(a,k)Anonymous scheme for privacy-preserving data collection in IoT-based healthcare services systems," Journal of Medical Systems, vol. 42, no. 3, pp. 56-59, 2018.

[14] R. Roshania, M. Mallow, N. Dunbar et al., "Successful implementation of a multicountry clinical surveillance and data collection system for ebola virus disease in west africa: findings and lessons learned," Global Health: Science and Practice, vol. 4, no. 3, pp. 394-409, 2016.

[15] D. Hai and T. Hua, "Development of a tracking-based system for automated traffic data collection for roundabouts," Journal of Modern Transportation, vol. 25, no. 1, pp. 1-12, 2017.

[16] S. Duan and J. Chen, "Research on the relationship between sports media and the healthy development of football industry: a case study in Spain football club real madrid," Journal of Computational and Theoretical Nanoscience, vol. 13, no. 3, pp. 2187-2192, 2016.

[17] F. A. Reegu, M. O. Al-Khateeb, W. A. Zogaan et al., "Blockchain-based framework for interoperable electronic health record," Annals of the Romanian Society for Cell Biology, vol. 25, no. 3, pp. 6486-6495, 2021.

[18] C.-H. Wu, Y.-H. Yuan, and S.-B. Tsai, "Using the DEMATEL model to expose core causal items of LibQUAL for improving library service quality: from the perspective of big data," Soft Computing, vol. 24, no. 8, pp. 5729-5739, 2020.

[19] S. Hermes, T. Riasanow, E. K. Clemons, M. Böhm, and H. Krcmar, "The digital transformation of the healthcare industry: exploring the rise of emerging platform ecosystems and their influence on the role of patients," Business Research, vol. 13, no. 3, pp. 1033-1069, 2020.

[20] X. Chen, H. Zhu, D. Geng, W. Liu, R. Yang, and S. Li, "Merging RFID and blockchain technologies to accelerate big data medical research based on physiological signals," Journal of Healthcare Engineering, vol. 2020, no. 5, 17 pages, Article ID 2452683, 2020.

[21] P. Li, C. Xu, H. Jin et al., "ChainSDI: a software-defined infrastructure for regulation-compliant home-based healthcare services secured by blockchains," IEEE Systems Journal, vol. 14, no. 2, pp. 2042-2053, 2020.

[22] Z. Yu, Y. Liu, and C. Zhu, "Application of propofol in oral and maxillofacial surgery anesthesia based on smart medical blockchain technology," Journal of Healthcare Engineering, vol. 2021, no. 1, pp. 1-11, 2021.

[23] Y. Wang and D. Zeng, "Development of sports industry under the influence of COVID-19 epidemic situation based on big data," Journal of Intelligent \& Fuzzy Systems, vol. 39, no. 6, pp. 8867-8875, 2020.

[24] N. Domadiya and U. P. Rao, "Improving healthcare services using source anonymous scheme with privacy preserving distributed healthcare data collection and mining," Computing, vol. 103, no. 4, pp. 1-23, 2021. 